Polymer Journal, Vol. 25, No. 6, pp 553-559 (1993)

\title{
Measurement of Viscoelastic Properties of Polymer Solutions by Torsional Crystals
}

\author{
Haruyo YoshizAKI \\ Department of Materials Science and Engineering, \\ National Defense Academy, \\ 1-10-20, Hasahirimizu, Yokosuka 239, Japan
}

(Received July 20, 1992)

\begin{abstract}
The network analyzer was used for measurement of the viscoelastic properties of dilute polymer solutions. It is a sensitive instrument to measure resonance resistance and resonant frequency of crystals by the admittance circle diagrams shown on the digital oscilloscope. Measurements can be conducted more correctly and rapidly compared to the previous measuring system. Its validity was verified by measuring the complex shear modulus of dilute polymer solutions and comparing the results with the Rouse theory. The complex shear modulus was determined by measuring changes in the properties of a torsionally vibrating crystal. Frequency dependence of complex shear modulus is investigated in the frequency range from 3 to $252 \mathrm{kHz}$ and temperature range from -60 to $23^{\circ} \mathrm{C}$. The samples are several kinds of dilute solutions of polystyrene in toluene. The use of polystyrene is limited in narrow molecular weight fractions ranging in number-average molecular weight from $2.1 \times 10^{3}$ to $1.60 \times 10^{5}$. The results agreed with the theory by Rouse.

KEY WORDS Network Analyzer / Torsional Crystal / Polymer Solution /

Polystyrene / Rouse Theory /
\end{abstract}

In order to investigate the viscoelastic properties of polymer solutions, it is necessary to measure the complex shear modulus at various frequencies. If liquids are sheared sufficiently rapidly, they exhibit a shear elastic effect as well as viscous effect. ${ }^{1}$ It is the purpose of this paper to describe a method for measuring viscoelastic properties of polymer solutions at ultrasonic frequencies using torsionally vibrating crystals and a new measuring system, the network analyzer.

Measurement of viscoelastic properties of dilute polymer solutions requires very sensitive instruments. The first technique suitable for work in this field was introduced by Mason. ${ }^{1,2}$ Mason et al. measured the resistance and reactance of a torsional crystal by a bridge circuit. This method has been extended by McSkimin and his coworkers. ${ }^{3,4}$ They made improvement so that small change in phaseshift can be determined using AT-cut quartz unit. Birnboim and Ferry ${ }^{5}$ measured over a continuous frequency range from 0.01 to $5 \mathrm{~Hz}$ by amplitude-phase shift measurements. Wada and his coworkers ${ }^{6}$ measured amplitudes and phase-shift in the frequency range from 20 to $300 \mathrm{kHz}$.

In the present paper, a simple technique is presented for measuring accurately and directly the equivalent circuit elements of a crystal. The network analyzer used in this experiment is a fully automatic instrument designed to measure several impedance parameters of electronic devices. The procedure of measurement has been more simplified compared to that by using the previous electrical measurement systems.

The longitudinal relaxation is complicated even in a dilute solution due to the presence of excess absorption originated from a compressional mode motion. The merit of the shear measurement lies in the fact that relaxation behavior in a normal state is well 
known and analytic methods are firmly established. It is of interest to compare the theory with data on a polymer solution where long range cooperative motion plays an important role. Three narrow molecular weight fractions ranging in molecular weight from $2.1 \times 10^{3}$ to $1.6 \times 10^{5}$ were employed. The complex shear modulus of polystyrene-toluene solutions was measured at frequencies of $3 \mathrm{kHz}$, $51 \mathrm{kHz}$, and $252 \mathrm{kHz}$. The data are compared with the theoretical curves by Rouse. ${ }^{7}$

\section{EXPERIMENTAL}

\section{Principle of the Method}

If we set a crystal vibrating in a purely torsional mode, the motion is tangential to the surface and highly attenuating viscous waves can be set up in the medium.

The shear wave equation for a plane wave propagation along $z$ can be derived from the definition of viscosity. Thus the different equation becomes

$$
\rho \frac{\partial \xi}{\partial t}=\eta^{*} \frac{\partial^{2} \xi}{\partial z^{2}},
$$

where $\rho$ is the density of the medium, $\xi$ the velocity of the motion along $x$, and $\eta^{*}=$ $\left(G^{*} / i \omega\right)=\eta-i\left(G^{\prime} / \omega\right)$, complex shear viscosity, where $G^{*}=G^{\prime}+i G^{\prime \prime}$, complex rigidity, $\omega$ is the angular frequency. For simple harmonic motion, the plane viscous wave has the solution,

$$
\xi=\xi_{+} \mathrm{e}^{i\left(\omega t-k^{*} z\right)} .
$$

Inserting eq 2 into eq 1 , the plane viscous wave has the following relation,

$$
i k^{*}=\left(\frac{i \omega \rho}{\eta^{*}}\right)^{1 / 2} .
$$

At the surface of the crystal, $z=0$, the force acting on liquids is represented by the relation,

$$
F_{z=0}=-A \eta^{*}\left(\frac{\partial \xi}{\partial z}\right)_{0}=i A \xi_{0} \eta^{*} k^{*}
$$

The acoustic impedance, $Z_{\mathrm{m}}$ becomes

$$
\begin{aligned}
Z_{\mathrm{m}} & =F_{z=0} / A \xi_{0}=i \eta^{*} k^{*}=\left(i \omega \rho \eta^{*}\right)^{1 / 2} \\
& =R_{\mathrm{m}}+i X_{\mathrm{m}}
\end{aligned}
$$

where $R_{\mathrm{m}}$ and $X_{\mathrm{m}}$ are the specific acoustic resistance and reactance, respectively. Squaring the latter two terms of eq 5, we get the relations,

$$
\begin{aligned}
G^{\prime} & =\frac{R_{\mathrm{m}}{ }^{2}-X_{\mathrm{m}}{ }^{2}}{\rho}, \\
G^{\prime \prime} & =\frac{2 R_{\mathrm{m}} X_{\mathrm{m}}}{\rho} .
\end{aligned}
$$

The attenuation of wave propagation introduces a loading effect on the crystal which can be measured by increase in the resonant resistance and decrease of the resonant frequency of the crystal. The change in the measured electrical resistance $\Delta R_{\mathrm{E}}$ and lowering in frequency $\Delta f$ are related to $R_{\mathrm{M}}$ and $X_{\mathrm{M}}$ according to the formulae,

$$
\begin{aligned}
& R_{\mathrm{M}}=\frac{\Delta R_{\mathrm{E}}}{K_{1}}, \\
& X_{\mathrm{M}}=\frac{\Delta f}{K_{2}},
\end{aligned}
$$

where $K_{1}$ and $K_{2}$ are constants depending upon the geometrical and electrical characteristics of

Table I. Liquids for determination of constants, $K_{1}$ and $K_{2}$

\begin{tabular}{lc}
\hline \multicolumn{1}{c}{ Liquids } & $\sqrt{\rho \eta}$ at $23^{\circ} \mathrm{C} / \mathrm{g} \cdot \mathrm{cm}^{-2} \cdot \mathrm{s}^{-1 / 2}$ \\
\hline Ethyl ether & $4.06 \times 10^{-2}$ \\
$n$-Hexane & $4.54 \times 10^{-2}$ \\
Toluene & $7.00 \times 10^{-2}$ \\
Benzene & $7.40 \times 10^{-2}$ \\
Silicone & $8.10 \times 10^{-2}$ \\
Cyclohexane & $8.25 \times 10^{-2}$ \\
$n$-Butylbenzene & $9.17 \times 10^{-2}$ \\
Silicone & $9.29 \times 10^{-2}$ \\
Silicone & $1.32 \times 10^{-1}$ \\
Nitrobenzene & $1.48 \times 10^{-1}$ \\
Silicone & $1.54 \times 10^{-1}$ \\
Silicone & $2.65 \times 10^{-1}$ \\
Silicone & $2.97 \times 10^{-1}$ \\
Polybutene & $3.14 \times 10^{-1}$ \\
\end{tabular}


Table II. Properties of polystyrene investigated as solute

\begin{tabular}{cccrrrr}
\hline Smple No. & $\bar{M}_{n}{ }^{\mathrm{a}}$ & $\bar{M}_{w} / \bar{M}_{n}{ }^{\mathrm{b}}$ & \multicolumn{1}{c}{$\bar{Z}^{\mathrm{c}}$} & $N^{\mathrm{d}}$ & \multicolumn{1}{c}{$c / \mathrm{g} \mathrm{cm}^{-3 \mathrm{e}}$} & $C^{*} / \mathrm{g} \mathrm{cm}^{-3 \mathrm{f}}$ \\
\hline 1 & $1.60 \times 10^{5}$ & 1.06 & 1524 & 305 & $2.49 \times 10^{-2}$ & $6.70 \times 10^{-2}$ \\
2 & $1.60 \times 10^{5}$ & 1.06 & 1524 & 305 & $5.00 \times 10^{-2}$ & $6.70 \times 10^{-2}$ \\
3 & $1.00 \times 10^{4}$ & 1.10 & 95 & 19 & $1.00 \times 10^{-1}$ & $2.70 \times 10^{-1}$ \\
4 & $2.10 \times 10^{3}$ & 1.10 & 20 & 4 & $1.00 \times 10^{-1}$ & $5.89 \times 10^{-1}$ \\
\hline
\end{tabular}

a $\bar{M}_{n}$, the number-average molecular weight; $\bar{M}_{n}=M_{0} \times \bar{Z}$, where $M_{0}$ is the molecular weight of monomer, 105 .

b The ratio $\bar{M}_{w} / \bar{M}_{n}$, where $\bar{M}_{w}$ is the weight average molecular weight.

c $\bar{Z}$, the average degree of polymerization; $\bar{Z}=N q, q=5$.

d $N$, the number of Rouse mode.

e $c$, the concentration used in the experiment.

f $C^{*}$, the concentration estimated by eq 18 .

the quartz crystal and may be determined from measurements using a Newtonian liquid of known shear mechanical impedance. Suitable liquids used in this investigation are listed in Table I.

Two types of quartz crystals, fork crystal of $3 \mathrm{kHz}$ and cylindrical crystal of $51 \mathrm{kHz}$ and $252 \mathrm{kHz}$, were used in this investigation.

\section{Samples}

Commercial samples of polystyrene produced by Polymer Laboratories Ltd. were used. Their characteristics are listed in Table II. Toluene of the special grade by Tokyo Kasei Kogyo Co., Ltd. was used as a solvent.

Temperature was measured by the crystal thermometer (hp. 2801A). Density was measured by an improved picnometer. The static viscosity of polymer solutions and solvent were measured using Cannon-Fenske viscometers and improved Ubbelohde viscometers.

\section{Apparatus}

The network analyzer (ADVANTEST R4611) measures amplitude, phase, and impedance at extended frequencies ranging from $10 \mathrm{~Hz}$ to $300 \mathrm{MHz}$. It has a digital oscilloscope which shows digital data together with admittance circle diagrams.

When the crystal resonator is connected with the network analyzer, the equivalent circuit is shown in Figure 1. It is expressed by the parallel

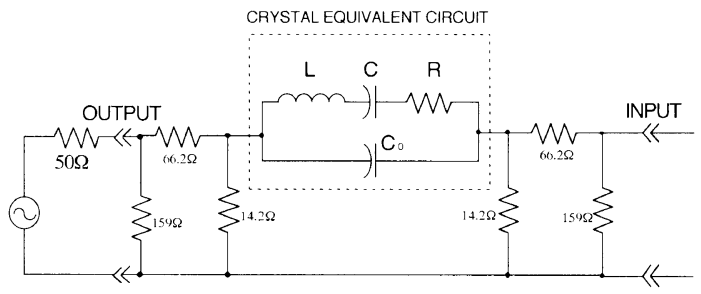

Figure 1. Equivalent circuit of the crystal connected with the network analyzer.

equivalent capacitance of free space $C_{0}$ and motional impedance of the crystal composed of capacitance $C$, inductance $L$, and resistance $R$. From Figure 1, we find that the admittance of crystal $Y_{\mathrm{c}}$, conductance $G_{\mathrm{c}}$, and susceptance $B_{\mathrm{c}}$ are written in the following equations. ${ }^{8}$

$$
\begin{aligned}
& Y_{\mathrm{c}}=i \omega C_{0}+\frac{1}{R+i \omega L+\frac{1}{i \omega C}}=G_{\mathrm{c}}-i B_{\mathrm{c}} \\
& G_{\mathrm{c}}=\frac{R}{R^{2}+\left(\omega L-\frac{1}{\omega C}\right)^{2}} \\
& B_{\mathrm{c}}=\omega C_{0}-\frac{\omega L-\frac{1}{\omega C}}{R^{2}+\left(\omega L-\frac{1}{\omega C}\right)^{2}}
\end{aligned}
$$

where $\omega$ is angular frequency.

From eq 11 and 12, a circular locus for representing admittance of crystals can be 


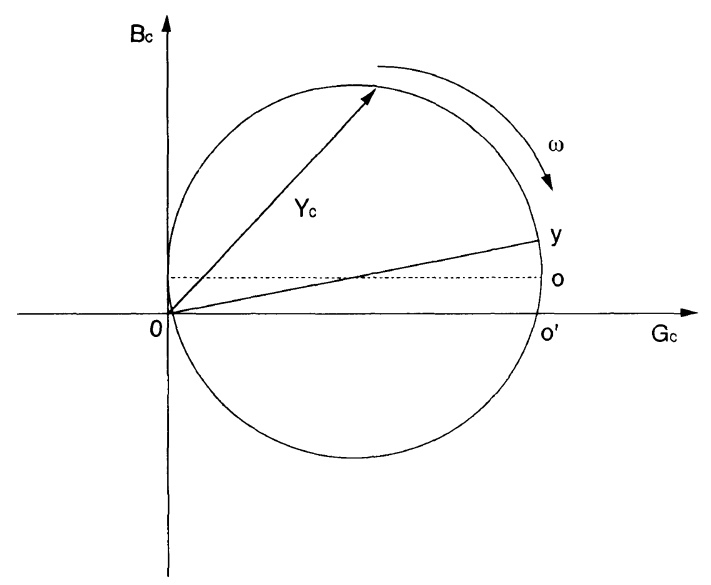

Figure 2. Admittance circle diagram of the crystal.

represented as shown in Figure 2. As the frequency increases, the operating point on the circle moves clockwise. The point $y$ shows the frequency of the total current or the minimum impedance. It is a slightly lower frequency than that at mechanical resonance. If the denominator of eq 11 becomes the minimum, the conductance goes to $1 / R$ at a frequency

$$
\omega_{1}=\sqrt{1 /(L C)}
$$

which is the resonance frequency of the mechanical series branch of the crystal. Thus, the frequency and resistance at resonance are determined by the maximum conductance of the admittance circle. Such desired parameters at the point $o$ can be given by digits through the front panel control keys. The point $o^{\prime}$ shows the electrical series resonance frequency.

\section{RESULTS AND DISCUSSION}

The temperature dependence of resonance frequency $f_{0}$ in dry air is expressed by the following experimental expressions for three crystals,

$$
\begin{aligned}
f_{0}= & 2.5226 \times 10^{5}+1.4349 t \\
& +6.6358 \times 10^{-3} t^{2} \\
f_{0}= & 5.1182 \times 10^{4}+0.21573 t
\end{aligned}
$$

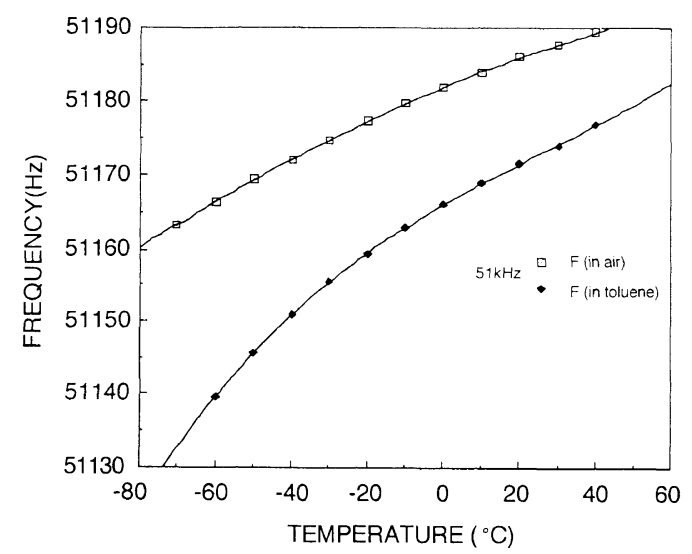

Figure 3. Resonance frequency plotted against temperature for the $51 \mathrm{kHz}$ crystal in air and in toluene.

$$
\begin{aligned}
& -7.2163 \times 10^{-4} t^{2} \\
f_{0}= & 2.8724 \times 10^{3}+5.5329 \times 10^{-2} t \\
& +5.2890 \times 10^{-4} t^{2}
\end{aligned}
$$

where $t$ represents temperature measured in degrees centigrade. They are obtained from the best fit curve of measured values by computer. Typical magnitudes of change of resonance frequency in dry air plotted against temperature appear in Figure 3 for $51 \mathrm{kHz}$ crystal.

Many kinds of Newtonian liquids shown in Table I were measured by the previous method. The value of $G^{\prime}$ becomes zero in simple Newtonian liquids. Then the following relations is obtained.

$$
R_{\mathrm{M}}=X_{\mathrm{M}}=\sqrt{\pi f \eta \rho}
$$

Thus, the constants $K_{1}$ and $K_{2}$ in eq 8 and 9 may be evaluated by carefully measuring $\Delta R_{\mathrm{E}}$ and $\Delta f$ on a liquid of accurately known density $\rho$ and viscosity $\eta$, at given frequency and given temperature. The temperature dependence of $K_{1}$ and $K_{2}$ is determined from that of $\Delta R_{\mathrm{E}}$ and $\Delta f$ of toluene.

Measurements by the method described above were conducted on all solutions shown in Table II. Typical results are shown in Figure 4 for sample 1 . The values of $G^{\prime}$ and $G^{\prime \prime}$ are plotted against frequency at several temperatures from 23 to $-60^{\circ} \mathrm{C}$. 


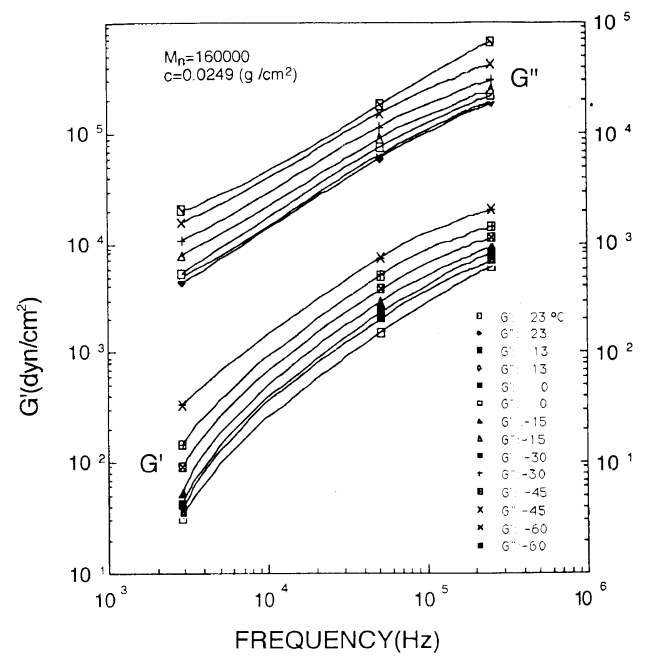

Figure 4. Measured values of $G^{\prime}$ and $G^{\prime \prime}$ plotted against frequency at several temperatures for the sample 1 .

Rouse $^{9}$ developed molecular theories of the viscoelastic properties of dilute solutions of polymers. When his theory is applied to the concentrated solution, the real and imaginary parts of the complex shear modulus $G^{*}$ at the normal state for a Gaussian distribution of molecular weight are given by

$$
\begin{aligned}
G_{0}{ }^{\prime}= & \frac{c R T}{\bar{M}_{n}} \sum_{p=1}^{N} \int_{0}^{\infty} \frac{\left(\omega \tau_{1}\right)^{2} \mu^{4} / p^{4}}{1+\left(\omega \tau_{1}\right)^{2} \mu^{4} / p^{4}} \mathrm{e}^{-\mu} \mathrm{d} \mu \\
G_{0}{ }^{\prime \prime}= & \omega \eta_{\mathrm{a}}+\frac{c R T}{\bar{M}_{n}} \sum_{p=1}^{N} \int_{0}^{\infty} \frac{\omega \tau_{1} \mu^{4} / p^{4}}{1+\left(\omega \tau_{1}\right)^{2} \mu^{2} / p^{4}} \\
& \times \mathrm{e}^{-\mu} \mathrm{d} \mu \\
\tau_{1}= & 3\left(\eta_{0}-\eta_{\mathrm{a}}\right) \bar{M}_{n} / \pi^{2} c R T
\end{aligned}
$$

where $c$ is concentration per ml., $R$ gas constant, $T$ absolute temperature, $\eta_{0}$ viscosity of polymer solution at the normal state, $\eta_{\mathrm{a}}$ solvent viscosity, and $\mu=\bar{M}_{w} / \bar{M}_{n}$. The number of Rouse modes $N$ and number of monomer units in a segment $q$ have the relation $N q=\bar{Z}$, where $\bar{Z}$ is average degree of polymerization. When the molecular weight of monomer unit and the number average molecular weight are denoted by $M_{0}$ and $\bar{M}_{n}$, respectively, the relation $\bar{Z} M_{0}=\bar{M}_{n}$ holds.

The value of $\tau_{1}$ can be calculated by eq 16
Table III. Parameters of the viscosity equation, $\ln \eta=A+B / T$

\begin{tabular}{ccc}
\hline Smple No. & $A$ & $B$ \\
\hline 1 & -8.009 & 1264 \\
2 & -7.329 & 1264 \\
3 & -8.858 & 1253 \\
4 & -9.121 & 1251 \\
\hline
\end{tabular}

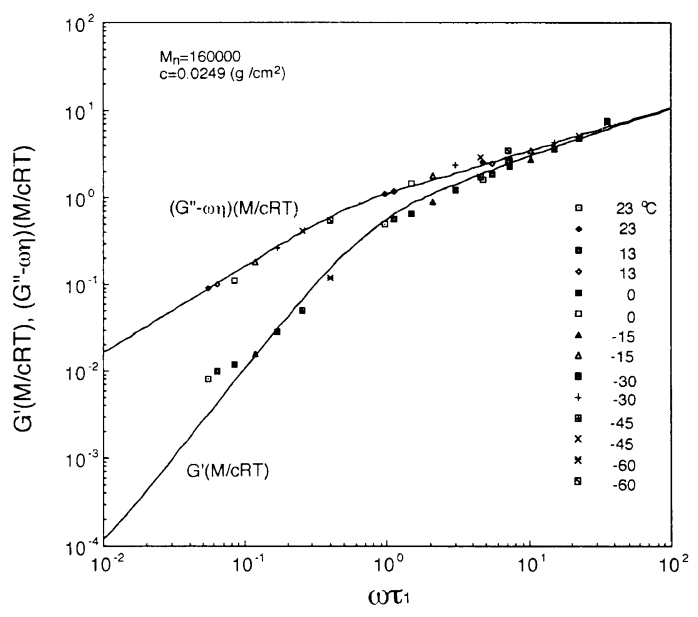

Figure 5. Reduced values of $G^{\prime}$ and $G^{\prime \prime}$ plotted against $\omega \tau_{1}$ for the sample 1 .

and measured values of $\eta_{0}$ and $\eta_{\mathrm{a}}$. The results of the static viscosity are expressed by Andrade's equation:

$$
\ln \eta_{0}=A+\frac{B}{T},
$$

where $A$ and $B$ are constants for given solution, whose values are determined by a digital computer to obtain a least-squares fit to the data, and given in Table III.

The reduced values for $G^{\prime}$ and $G^{\prime \prime}$ are plotted against $\omega \tau_{1}$ in Figure 5. The two solid curves represent the theoretical lines calculated from eq 14 and 15 when $N=305$. The theory agrees with the data of reduced $G^{\prime}$ and $G^{\prime \prime}$ in a range of $\omega \tau_{1}$ from $\sim 5 \times 10^{-2}$ to $\sim 5 \times 10^{1}$. The deviation of the measured values of $G^{\prime} /(c R T)$ at $\omega \tau_{1}$ below $10^{-1}$ seems to be due to the roughness of $3 \mathrm{kHz}$ crystal. 


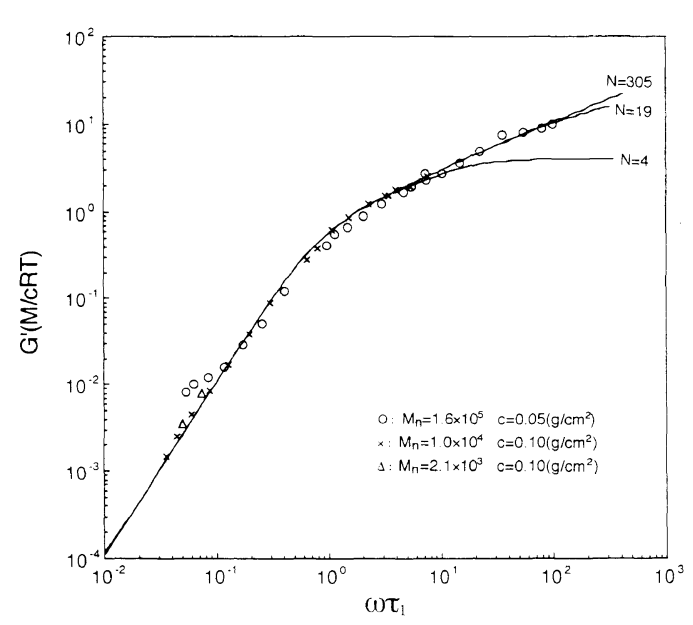

Figure 6. Reduced values of $G^{\prime}$ plotted against $\omega \tau_{1}$ for the sample $2-4$. The marks of $O, \times$, and $\triangle$ represent the measured values of the sample $2-4$, respectively. The solid lines are theoretical curves by Rouse of $N=305,19$, and 4 , respectively.

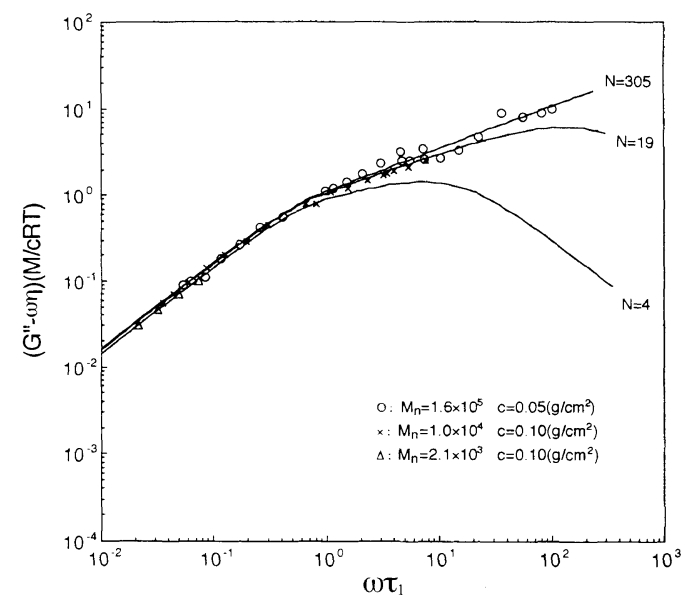

Figure 7. Reduced values of $G^{\prime \prime}$ plotted against $\omega \tau_{1}$ for samples 2-4. The marks of $O, \times$, and $\triangle$ and the solid lines have the same meaning as Figure 6.

The reduced $G^{\prime}$ and $G^{\prime \prime}$ for sample 2-4 are plotted against $\omega \tau_{1}$ as shown in Figures 6 and 7. The marks $\bigcirc, \times$, and $\triangle$ represent measured values of samples $2-4$, respectively. The behaviors of samples $2-4$ do not coincide with the only single theoretical curve at higher values of $\omega \tau_{1}$. It is necessary to choose a suitable number of Rouse modes according to mo- lecular weight. The solid lines are the theoretical curves by Rouse of $N=305,19$, and 4 , respectively. The Rouse mode numbers correspond to $q=5$ for any molecular weight. Thus the measured values are at least a good first approximation to the Rouse theory when $q=5$.

Agreement between theory and experiment is to be expected as the result of the following: (1) measurement at low frequencies where the spectrum by the Rouse theory is dominant, (2) monodispersity of a sample as shown in Table II, and (3) dilute solution where free-draining phenomena can be observed.

Lamb et al. ${ }^{10}$ made the same measurement for the molecular weight fraction with $2.39 \times 10^{5}$ and above. They predicted good agreement with the theory of Zimm. Some results of Rouse and Sittel $^{7}$ for polystyrene $f$ molecular weight $2.53 \times 10^{5}$ agree with the Rouse theory. Tshoegl and Ferry ${ }^{11}$ also found that in a solvent of high viscosity a polystyrene of molecular weight $1.7 \times 10^{6}$ agrees with the Rouse theory. The essential difference between the theories by Rouse and Zimm lies in the type of interaction between the polymer molecule and flowing solvent. Rouse assumes that the velocity of the solvent flowing through a molecule is unaffected by the presence of the molecule (free-draining case), while Zimm assumes that solvent velocity is less in the region of the center of the molecule than at its outer fringes (nonfree-draining case). The frictional coefficient for the molecule should depend on the size of segment, the number of segments, the size of the molecule and concentration.

If the size of segment $b$ and number of segments $N$ progressively decreased, the solvent flow will penetrate closer to the center and lead eventually to free-draining. The values of $b$ and $N$ have the same effect on the frictional properties of polymer solutions, if they are independent of each other. A decrease in $N$ will have the same effect as an increase in $b$, if the molecular weight is held constant. The 
contradiction of the effects of $b$ and $N$ may be concerned with the value of $q$. The magnitudes of molecular weight measured in this experiment are very small compared to that by Lamb. As predicted by Bueche, ${ }^{12}$ it is supposed that the molecule does not behave like a random coil, but essentially like a rod.

If the size of molecule or the radius of gyration $\sqrt{\overline{s^{2}}}$ is represented by the root-meansquare distance of segment from the center gravity, the critical concentration where molecules contact with each other in polymer solutions should be defined as ${ }^{13}$

$$
C^{*}=\frac{M}{\frac{4}{3} \pi\left(\sqrt{\overline{s^{2}}}\right)^{3} N_{\mathrm{A}}}
$$

where $N_{\mathrm{A}}$ is Avogadro number. By statistical treatment when $N \gg 1$, the following relations is derived,

$$
\sqrt{\overline{s^{2}}}=\sqrt{r^{2} / 6}
$$

where $\overline{r^{2}}$ is the mean-square end-to-end distance. Since the relation $\left(\overline{r^{2}} / M\right)^{1 / 2}=0.602$ has been obtained, ${ }^{12,14}\left(\overline{s^{2}}\right)=0.602 \sqrt{M / 6}$ will be derived. Putting this value into eq 18 , the values of $C^{*}$ are obtained as shown in Table II. The values of $C^{*}$ are also obtained from the relation $C^{*}[\eta]=1.67^{13}$ and the reference values of $[\eta] .{ }^{14}$ Both are nearly equal. Comparing $C^{*}$ with $c$, it is reasonable to consider that samples $1-4$ are dilute solutions.

The viscoelasticity of solutions of polystyrene in toluene can be explained by Rouse theory, when the molecular weight is smaller than $\sim 10^{5}$ and the concentration is so small as to be considered as a dilute solution.

\section{CONCLUSIONS}

The network analyzer is a useful and sensible instrument to measure the viscoelasticity of polymer solutions precisely using torsional crystal. From the results it is found that the complex shear modulus of polystyrene in toluene can be explained by Rouse theory, when the concentration is smaller than the critical solution and the values of $\omega \tau_{1}$ are below $10^{2}$.

Acknowledgment. The author wishes to express her gratitude to Professor Shoso Dohi of the National Defense Academy for his encouragement throughout this work.

\section{REFERENCES}

1. W. P. Mason and J. N. Murray Hill, Trans. A.S.M.E., 69, 359 (1947).

2. W. P. Mason, W. O. Baker, H.J. McSkimin, and J. H. Heiss, Phys. Rev., 75, 836 (1949).

3. H. J. McSkimin and P. Andreatch, Jr., J. Acoust. Soc. Am., 42, 248 (1967).

4. R. S. Moore, H. J. McSkimin, C. Gieniewski, and P. Andreatch, Jr., J. Chem. Phys., 47, 3 (1967).

5. Meyer H. Birnboim and John D. Ferry, J. Appl. Phys., 32, 2305 (1961).

6. H. Nakajima and Y. Wada, Polym. J., 1, 727 (1970).

7. P. E. Rouse, Jr. and K. Sittel, J. Appl. Phys., 24, 690 (1953).

8. S. Yamamoto, S. Sugawara, Y. Tomikawa, and M. Konno, Nihon Onkyogaku Kaishi, 34, 455 (1978).

9. P. E. Rouse, Jr., J. Chem. Phys., 21, 1272 (1953).

10. G. Harrison, J. Lamb, and A. J. Matheson, J. Phys. Chem., 68, 1072 (1964).

11. Richard B. De Mallie, Jr., Meyer H. Birnboim, J. E. Frederick, N. W. Tschoegle, and John D. Ferry, J. Phys. Chem., 66, 536 (1962).

12. F. Buche, "Kobunshi no Bussei (Physical Properties of Polymers)," Asakura Publishing Co., Ltd., Tokyo, 1970, p 16

13. K. Ogino, Kobunshi, 32, 808 (1983).

14. "Polymer Handbook," 3rd ed, Vol. 5, J. Brandrup, Hoechest AG, and E. H. Immergut, Ed., John Wiley \& Sons, New York, N.Y., 1989. 